fluvio-hydrological and geospatial techniques. SN Appl. Sci., 2019, 1(5), 494; https://doi.org/10.1007/s42452-019-0516-2.

15. Dunn, J. A., Geology and petrology of Eastern Singhbhum and surrounding areas. Mem. Geol. Surv. India, 1942, 69, 261-456; https://ci.nii.ac.jp/naid/20000875781/\#cit

16. Roy, A. B., Indian Shield: insight into the pristine size, shape and tectonic framework. Indian J. Geosci., 2012, 66, 181-192.

17. Pascoe, E. D., A Manual of Geology of India and Burma, Geological Survey of India, Calcutta, 1973, vol. 1, p. 485.

18. Roy, A. B., Dutt, K. and Rathore, S., Development of ductile shear zones during diapiric magmatism of nepheline syenite and exhumation of granulites, examples from central Rajasthan, India. Curr. Sci., 2016, 110, 1094-1101; doi:10.18520/cs/v110/i6/10941101.

19. Singh, Y. and Krishna, V., Rb-Sr geochronology and petrogenesis of Granitoids from the Chotanagpur Granite Gneiss Complex of Raikera-Kunkuri Region, Central India. J. Geol. Soc. India, 2009, 74, 200-208; https://doi.org/10.1007/s12594-009-0122-9.

20. Saxena, V. P., Krishnamurthy, P., Murugan, C. and Sabot, H. K., Geochemistry of the granitoids from the central Surguja shear zone, India: geological evolution and implication on uranium mineralization and exploration. Explor. Res. Atom. Min., 1992, 5, $27-40$.

21. Mahadevan, T. M., Geological evolution of the Chotanagpur Gneiss Complex in a part of Purulia district, West Bengal. Indian J. Geol., 1992, 64, 1-22.

22. Mahadevan, T. M., Geology of Bihar and Jharkhand. Geological Society of India, Bangalore, 2002, p. 563.

23. Ghosh, N. C., Chatterjee, N., Mukherjee, D., Kent, R. W. and Saunders, A. D., Mineralogy and geochemistry of the Bengal Anorthosite Massif in the Chotanagpur Gneissic Complex at the Eastern Indian Shield Margin. J. Geol. Soc. India, 2008, 72, 263277.

24. Bhattacharya, P. K. and Mukherjee, S., Granulites in and around the Bengal anorthosite, eastern India; genesis of coronal garnet, and evolution of the granulite-anorthosite complex. Geol. Mag., 1987, 12, 21-32; https://doi.org/10.1017/S0016756800015752.

25. WHO, Iron deficiency anaemia: assessment, prevention and control, a guide for programme managers. World Health Organization, Geneva, 2001, pp. 47-62.

26. Fawell, J., Bailey, K., Chilton, J., Dahi, E. and Magara, Y., Fluoride in drinking-water, IWA Publishing, 2006; https://books. google.co.in/books?hl=en\&

27. Banerjee, G., Roy, P. K., Majumdar, A., Pal, S. and Mazumdar, A., A GIS Based Multi Criteria Evolution Technique (MCET) for Identifying Water Intake Construction Site (S) and Technology at Purulia district. In West Bengal Conference, in India Water Work Association 47th Annual Convention, Kolkata, 2015.

ACKNOWLEDGMENTS. We thank Public Health and Engineering, Govt of West Bengal, Geological Survey of India and the local people of Rarh Bengal for providing the valuable information and laboratory assistance.

Received 7 September 2020; revised accepted 29 December 2020

doi: $10.18520 / \mathrm{cs} / \mathrm{v} 120 / \mathrm{i} 7 / 1225-1233$

\section{Full-parameter optimization to locate multi-passage-seepage in abutment using groundwater temperature}

\author{
Xinjian Wang ${ }^{1, *}$ and Wei Wang ${ }^{2}$ \\ ${ }^{1}$ North China University of Water Resources and Electric Power, \\ Zhengzhou, 450011, China \\ ${ }^{2}$ Henan Geology and Mineral Construction Engineering Group Co \\ LTD, Zhengzhou, 450007, China
}

With groundwater temperature, hybrid-genetic algorithm is employed to locate multi-passage concentrated seepage underground to increase the probability of optimal global solutions, calculation efficiency and precision. The parameters of concentrated seepage passages (CSPs) indicated initially by the previous optimization and attraction basins of modified temperature residuals are evaluated again by the proposed full parameter optimization. The smaller CSP impacts on the stronger are eliminated, since all the parameters associated with all the CSPs are calculated by the last one-off optimization. In this case, three optimization steps are implemented with crossover fractions of $0.8,0.5$ and $0.45(0.3)$, and the modified resultant residuals are $13.441,2.27$ and 0.7 individually. Results of this method are more effective compared to those from other methods and actual applications.

Keywords: Abutment, dam safety, hybrid-genetic algorithm, geothermal temperature, seepage.

To effectively repair abutments and dams which are scoured by concentrated seepage passages (CSPs), the seepage locations need to be identified. Using underground temperature for dam leakage detection is a relatively fast, more environment-friendly as well as practical approach $^{1}$. Some studies indicate that this method is becoming fully independent and quantitative $e^{2-8}$. The lowest temperature method for location detection has been proposed and adopted earlier ${ }^{9,10}$.

Based on the principle of heat conservation and thermal equilibrium, analytical solutions to flux and single CSP location in the field of initial homogeneous temperature are calculated numerically ${ }^{6}$. Parameters representing CSP characteristics are estimated following the temperature data calibration with vertical thermal gradient ${ }^{11}$. Theoretical models and objective functions are established for planar CSP detection ${ }^{12}$. The number and initial locations of the CSPs are obtained by analysing spline interpolation curves of the temperature data or their residuals, and an inverse technique is applied for multi-CSP location detection ${ }^{1}$. A global optimization, composed of simulated annealing (SA) or genetic algorithm (GA) and multi-start (MS), is employed to estimate

*For correspondence. (e-mail: wangxinjian2009@yahoo.com) 
the locations of $\mathrm{CSPs}^{13}$. The sub-step optimization adopted in these studies fixes parameter values from the previous step and does not take into account minor CSP influence on major CSPs.

In this study, several methods, including narrowing the variable range via attraction basins (ABs), parallel computation, analysing and selecting the right crossoverfractions, hybrid algorithm and sub-step optimization for the CSP initial locations are adopted. Parameters of CSPs, identified by the previous optimization and ABs of modified temperature residuals are substituted into the objective function as unknown variables for the following optimization, called full parameter optimization. Thus, all the parameters passed down from previous optimizations are estimated again by the last step to eliminate the impact of weak CSPs on the strong ones.

Integrating a local search method with GA can help overcome most obstacles from finite population size, provide a better solution, improve the exploiting ability without limiting exploration of the search algorithm and speed up convergence to exact global optimum.

In a site with CSPs, exploration axes are set approximately vertical to the groundwater flow direction recognized by a geological investigation. Temperature detection holes are drilled vertically at appropriate distances. Temperature data collected at distances along vertical holes are calibrated with a geothermal gradient from which the recharge style and lowest temperature plane are identified. A modified temperature graph is achieved by moving the whole curve up for the recharge type of low groundwater temperature, where the temperature abnormality implies potential CSPs ${ }^{1}$.

Based on the lowest temperature and modified temperature residual characteristic method ${ }^{14}$, the objective function for GA is established as

$$
f=\| \text { error }_{i}-\max \left[\text { error }_{i}\right] \|_{2}^{2}
$$

where $f$ is the objective function in an optimization, max returns the maximum, $\|\bullet\|_{2}$ implies a two-norm. error ${ }_{i}$ represents the residual temperature vector (temperature for the first optimization) expressed as follows for oneoff optimization ${ }^{6}$

$$
\text { error }_{i}=T_{i}-\beta_{k 1}-\sum_{j=1}^{n+1}\left[\beta_{k, j+1} \times \ln \sqrt{\left(x_{i}-x_{0 j}\right)^{2}}\right]
$$

where $T_{i}$ denotes the temperature datum at detection point $i, k$ the $k$ th optimization, $n$ and $l$ are the CSP numbers from direct iteration and residual analysis of $(k-1)$ optimization respectively, $\beta$ s are coefficients and $x_{i}$ and $x_{0 j}$ are coordinates of the $i$ th temperature detection point and $j$ th CSP location.

In the hybrid optimization algorithm for CSP detection, Fmincon ${ }^{15}$, finding the minimum of a constrained non- linear multivariable function is implemented following GA. The minimum of a problem is specified by eq. (3)

$$
\min _{x} f^{\prime}(x) \text { such that }\left\{\begin{array}{l}
c(x) \leq 0 \\
A \cdot x \leq b \\
\mathrm{lb} \leq x \leq \mathrm{ub}
\end{array},\right.
$$

where $x$ is the independent variable representing detection locations and $f^{\prime}(x)$ an objective function for 'Fmincon' relayed from GA, returns a scalar. Function $c(\mathrm{x})$ and matrix $A$ together with vector $b$ denote the constraint conditions between $x$ that include several components; while $\mathrm{lb}$ and $\mathrm{ub}$ the lower and upper bounds respectively.

Parameter values that control the computation process are assigned in four ways: (1) Conventional parameters such as population type, initial score, population range, selection function, elite count and migration interval are set based on the literature ${ }^{16-18}$. (2) Crossover fractions are studied prior to each optimization step. (3) Trials determine parameter values of the stopping criteria. (4) The others are set by default.

The initial guesses of parameters associated with CSP characteristics significantly impact the optimization effectiveness and duration. Sub-step residual analysis is employed to search initial CSP locations within ABs formed by temperature and/or residual curves, and the limits of the $\mathrm{AB}$ are set as the bounds. Some initial values are directly from previous optimization results, and others are from the $\mathrm{AB}$ number of initial temperature or its residuals.

In general, coordinates of the bottoms of ABs are set as initial CSP locations. If a bottom exactly corresponds to a detection point, the initial value is set near, but not at the point. Constraint bounds to a CSP location are determined according to the scope and symmetry of the corresponding AB. Trials and experiences initialize other coefficients. Table 1 lists the parameter settings.

The computation procedure divided into three steps and presented as follows:

(a) According to the lowest initial temperature curve, the number, initial locations, abnormality extent and relative abnormality of $\mathrm{ABs}$ are determined. The relation between objective function values and crossover fractions is studied at a step of 0.05 , and the crossover fraction corresponding to the smallest and stable function value is adopted. Initial values are assigned to associated parameters and constraint bounds in the objective function.

(b) After hybrid GA is performed with previous settings, the temperature residual mean, $\operatorname{error}_{i} / i$, is calculated. If the mean is not more than the detection precision (DP), the computation is completed. The values of parameter $x_{0 i}$ are deemed as the CSP locations when the objective function is minimum. 
Otherwise, values of $x_{0 i}$ are substituted into eq. (2) to obtain a modified residual vector $x e_{i}$

$$
x e_{i}=\text { error }_{i}-\max \left[\text { error }_{i}\right]
$$

The total CSP number and constraint bounds are determined by the previous optimization and distribution of the corresponding modified residual $x e$. After the right crossover fraction is determined, all the initial values and control computation parameters are substituted into eq. (2) to give a new objective function for the next optimization.

(c) Step (b) is repeated until the residual mean meets the stopping criteria.

This method is applied to an engineering project, a reservoir dam-abutment, to verify its effectiveness. Conditions and issues of the abutment, the distribution of the geology boreholes arrangement and the lowest ground temperature data are listed in Wang and Pan ${ }^{1}$. The detection axis is appropriately perpendicular to the groundwater flow as illustrated in the document 1.

Groundwater recharge of the dam-abutment is lowtemperature type $\mathrm{e}^{10}$. The distribution of the lowest temperature data illustrates only one $\mathrm{AB}$, of which the bottom coordinate is about $50 \mathrm{~m}$ as the initial location of the first CSP. The AB range is anywhere from 0 to $90 \mathrm{~m}$ as the lower and upper bounds respectively. The objective function for inverse analysis is as in eq. (2), where $k=0$; $l=1$, and there are three parameters for optimization.

Table 1. Conventional parameter settings

\begin{tabular}{ll}
\hline Parameters & \multicolumn{1}{c}{ Value/setting } \\
\hline $\begin{array}{l}\text { Population } \\
\text { Type }\end{array}$ & Double vector \\
Size & 8000 \\
Creation function & Uniform \\
Initial population & Constraint dependent \\
Initial range & {$[-10,10]$} \\
Migration & \\
Fraction & 0.2 \\
Direction & Both \\
Interval & 20 \\
Constraint & \\
Initial penalty & 10 \\
Penalty factor & 100 \\
Fitness scaling function & Rank \\
Selection function & Stochastic uniform \\
Reproduction elite count & $0.05^{*}$ Population size \\
Stop criterial & \\
Generations & 500 \\
Time limit & $\infty$ \\
Fitness limit & $-\infty$ \\
Stall generations & 50 \\
Stall test & Average change \\
Function tolerance & $10^{-6}$ \\
Constraint tolerance & $1 \times 10^{-6}$ \\
Stall time limit & $\infty$ \\
\hline & \\
&
\end{tabular}

According to Figure $1 a$, the function falls at two values, 17.0 and 13.4, and the larger value is reached at five points of crossover fractions. All crossover fraction values corresponding to the large objective-function value and only one to the small are isolated. The longest segment of the continuous crossover fractions corresponding to the lowest function value centres at 0.8 between 0.65 and 0.95 , which produces relatively stable lowest function value. Therefore, 0.8 is adopted in this optimization.

Table 2 lists the other settings and iteration results. Successful convergence course is demonstrated in Figure $2 a$, and the middle optimization is specially enlarged to illustrate the convergence details. Generation diversity is noticeable. The mean value aggregates quickly from $6.09 \times 10^{4}$ to 5441 just through 10 generations, then to 345.4 through 20 generations, but slows down over later generations. The mean value changes slightly between 13.445 and 13.443 , in the middle of which the curve shows a gradual and gentle incline. The ultimate objective-function and tolerance values denote adequate convergence.

The first location estimated as $60.829 \mathrm{~m}$ and impacted by other CSPs is farther from detection point \#10 than those of 38.16 and $38.65 \mathrm{~m}$ by $\mathrm{SA}^{1,15}$, MS and $\mathrm{GS}^{16}$. The value of the objective function is less than those by SA (17.04), GS (17.03) and constraint nonlinear minimization (19.30), but larger than that by MS (10.63).

The mean objective function, modified temperature residual divided by detection point number, is larger than DP $\left(0.1^{\circ}\right)$, which indicates that new CSPs exist and need to be located by next optimization. Initial values for the next objective function are determined by the modified residual abnormality from this optimization. The modified residual distribution implies only one $\mathrm{AB}$ denotes a new CSP, and its bottom is near $180 \mathrm{~m}$ from detection point \#10.

Considering a new CSP from the analysis above, $k, n$ and $l$ equal to 1 are substituted in the second objective function, in which error ${ }_{i}$ is expressed as

$$
\text { error }_{i}=T_{i}-\beta_{11}-\beta_{12} \times \ln \left|x_{i}-x_{01}\right|-\beta_{13} \times \ln \left|x_{i}-x_{02}\right| \text {, }
$$

where five parameters are expected to be optimized.

It can be seen from Figure $1 b$ that the new function depending on crossover fractions falls at six values. The two biggest objective-function values are significantly

Table 2. First optimized leakage location by hybrid-GA

\begin{tabular}{lcccc}
\hline Parameters & $\beta_{01}$ & $\beta_{02}$ & $x_{01}$ & $\begin{array}{c}\text { Objective } \\
\text { function value }\end{array}$ \\
\hline $\begin{array}{l}\text { Iteration result } \\
\text { Lower and upper } \\
\text { bounds }\end{array}$ & 2.637 & 2.955 & 60.829 & 13.441 \\
\hline 0,20$]$ & {$[0,30]$} & {$[20,90]$} & \\
\hline
\end{tabular}


(a)

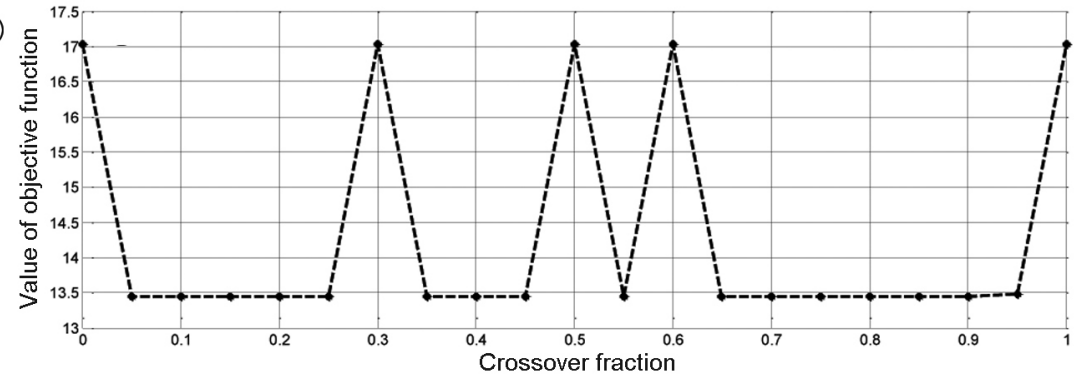

(b)

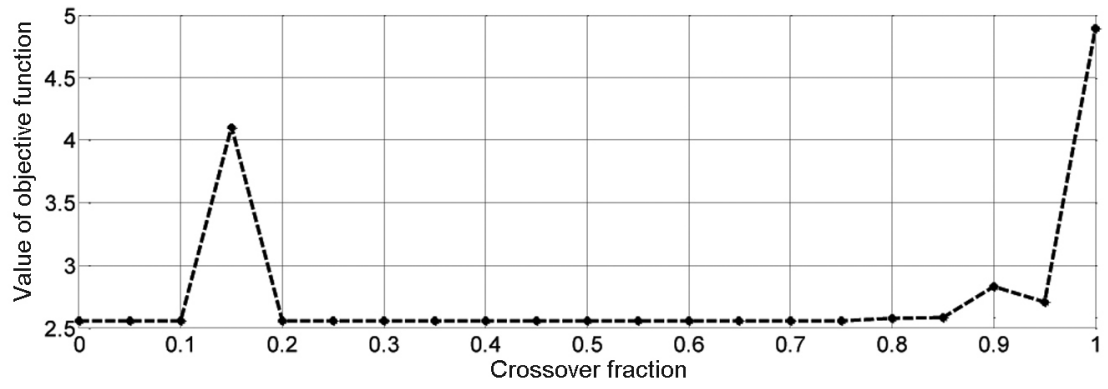

(c)
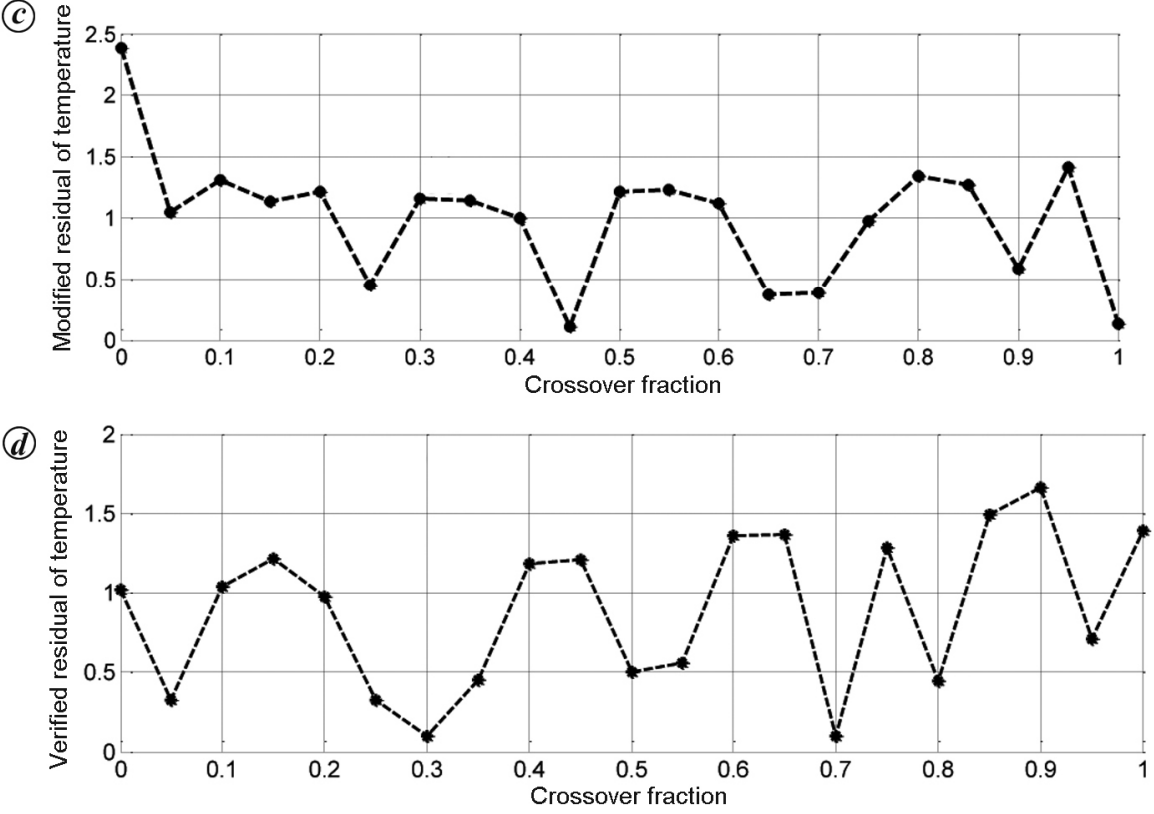

Figure 1. Relation between values of objective function and crossover fractions of each optimization: $\boldsymbol{a}$, first optimization; $\boldsymbol{b}$, second optimization; $\boldsymbol{c}$, four CSPs in the third optimization; $\boldsymbol{d}$, five CSPs in the third optimization.

larger than others. The longest stretch of continuous lowest values is from 0.2 to 0.75 , of which the centre 0.5 is selected as the crossover fraction in the second optimization.

Linear constraint condition is added to this optimization for two CSPs with different seepage intensities. The specific relationship between the two CSPs is determined by the relative abnormal magnitudes of the two ABs. Other settings are identical to those in the previous optimization. Table 3 lists some of them, together with optimization results.

As shown in Figure $2 b$, the second optimization successfully converges. The generation diversity is good in that the largest mean is as large as $8.78 \times 10^{4}$, slightly larger than that of the first optimization. The mean aggregates quickly to 7018 over four generations and to 1099 over eight generations, then shows slow variation as it gets smaller over many generations. The middle iteration indicates that the mean fluctuates at inconsistent amplitudes between about 300 and 600 over most of the duration. The mean and distance are greatly larger than those of the first optimization, but their general processes are similar.

The objective function value (2.27) corresponding to the best individual is distinctly less than 13.441 from the first optimization. The seepage intensity of the second 

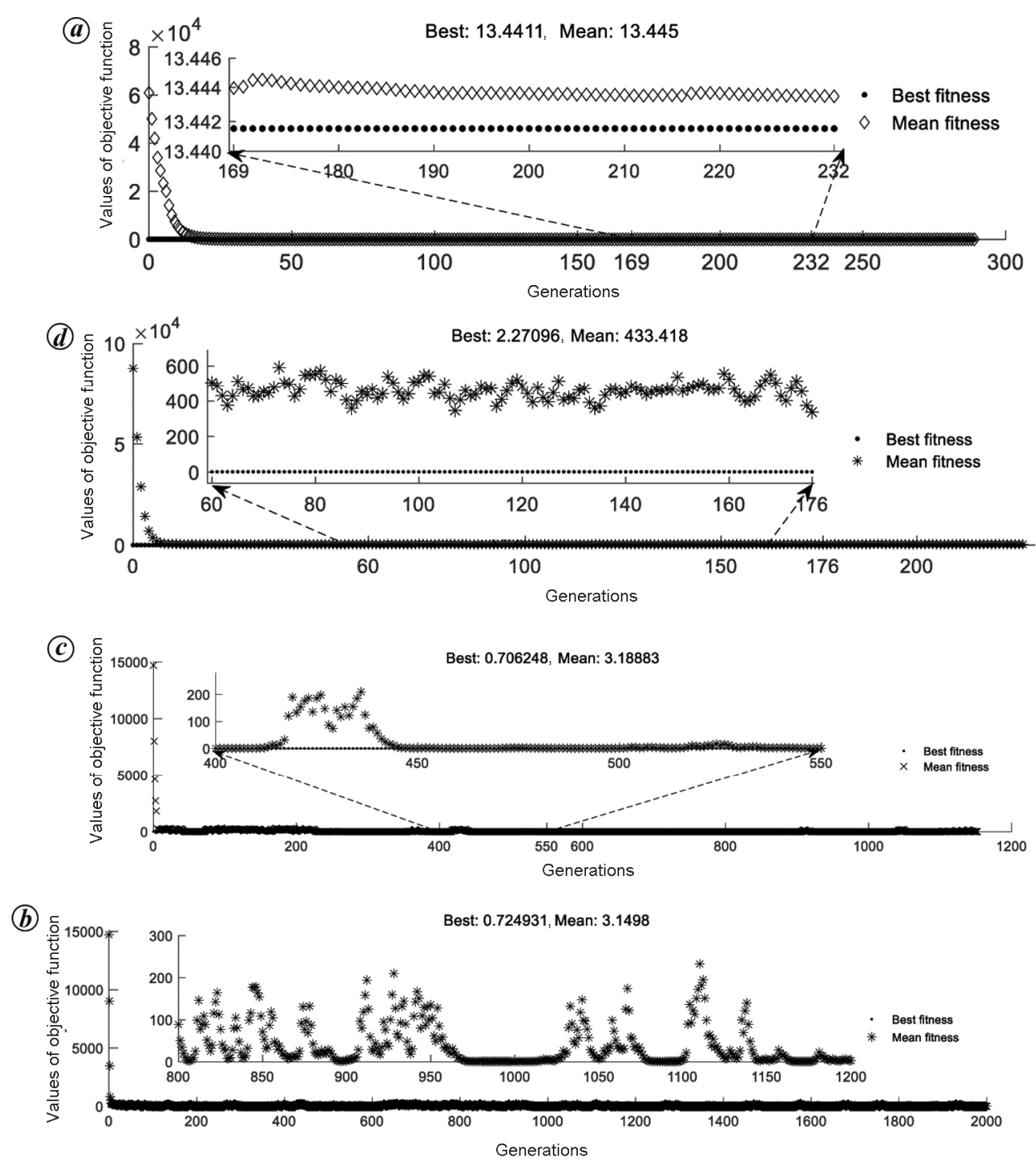

Figure 2. Converge process and best individuals of each optimization: $\boldsymbol{a}$, first optimization; $\boldsymbol{b}$, second optimization; $\boldsymbol{c}$, four CSPs in the third optimization; $\boldsymbol{d}$, five CSPs in the third optimization.

Table 3. Settings and results of the second optimization

\begin{tabular}{lccccc}
\hline Parameters & $\beta_{11}$ & $\beta_{12}$ & $x_{01}$ & $\beta_{13}$ & $x_{02}$ \\
\hline Results & $2.6 \times 10^{-3}$ & 2.613 & 41.256 & 0.841 & 172.587 \\
Initial scope & {$[0,20]$} & {$[0,20]$} & {$[30,80]$} & {$[0,20]$} & {$[150,190]$} \\
Computation parameters & Objective function & Generations & Function account & Population & Function tolerance \\
Set or back values & 2.271 & 227 & 2280086 & 10000 & $1 \times 10^{-10}$ \\
\hline
\end{tabular}

CSP is weaker than that of the first, because $\beta_{13}$ is much less than $\beta_{12}$. The first CSP location from this optimization is less than that from the first, demonstrating that the second CSP has a large impact on the first location.

The modified residual distribution after the second optimization illustrates graphically three intact ABs with different abnormal magnitudes. The modified residuals vary from 0 to -1.2 , the mean of which is still larger than DP $\left(0.1^{\circ}\right)$, though much less than that in the first optimi- zation. Therefore, the next optimization needs to be performed. Three intact ABs appear with bottoms near 140, 230 and $340 \mathrm{~m}$. The new initial locations and constraints are set for the third optimization according to the bottom locations, abnormal ranges and relative magnitudes of ABs.

The modified residual from the second optimization at detection point \# 2 is close to DP. So this abnormality may or may not arise from the actual CSP. Two situations 
RESEARCH COMMUNICATIONS

\begin{tabular}{|c|c|c|c|c|c|c|c|}
\hline Parameters & $\beta_{21}$ & $\beta_{22}$ & $x_{01}$ & $\beta_{23}$ & $x_{02}$ & $\beta_{24}$ & $x_{03}$ \\
\hline Result 1 & $2.465 \times 10^{-4}$ & 2.730 & 39.791 & 0.436 & 179.491 & 0.242 & 139.565 \\
\hline Result 2 & $1.053 \times 10^{-3}$ & 2.713 & 39.861 & 0.453 & 179.374 & 0.250 & 139.435 \\
\hline Bounds & {$[0,20]$} & {$[0,20]$} & {$[30,80]$} & {$[0,20]$} & {$[90,240]$} & {$[0,20]$} & {$[80,170]$} \\
\hline Parameters & $\beta_{25}$ & $x_{04}$ & $\beta_{26}$ & $x_{05}$ & Objective function value & Generations & Population \\
\hline Result 1 & 0.0301 & 220.000 & & & 0.706 & 1152 & $400 \times 9$ \\
\hline Result 2 & 0.0219 & 220.000 & $6.57 \times 10^{-3}$ & 345.674 & 0.428 & 2213 & $400 \times 11$ \\
\hline Bounds & {$[0,20]$} & {$[200,280]$} & $\begin{array}{l}\text { Function } \\
\text { accounts }\end{array}$ & $\begin{array}{c}461671 \\
\text { (Result 1) }\end{array}$ & $\begin{array}{c}600359 \\
\text { (Result 2) }\end{array}$ & $\begin{array}{l}\text { Function } \\
\text { tolerance }\end{array}$ & $1 \times 10^{-6}$ \\
\hline
\end{tabular}

are considered for the third optimization to compare the results.

For the situation ignoring the minimum $\mathrm{AB}$ between 300 and $380 \mathrm{~m}$, the parameters $n, l$ and $k$ equal to 2 are substituted in eq. (2) to give a new objective function. Nine variables need to be estimated by the third optimization.

Figure $1 c$ shows the relation between the new objective function and crossover fractions. The new function obtains several different values, of which the largest is near 2.5 and far from others on the vertical coordinate. Most of the values are between 1.5 and 0.4 , but two points have the smallest function value and are isolated. The value corresponding to the crossover fraction of 0.45 is unstable compared with the previous value (Figure $1 \mathrm{~b}$ ). After several computational trials, it was found better to select 0.45 as the crossover fraction.

Figure $2 c$ shows the convergence process for the best individual, mean and final variable values for four CSP situations. The generation diversity is also good in that the largest mean, $1.47 \times 10^{4}$, has the same order of magnitude as those in previous optimizations. The mean in the middle iteration fluctuates inconsistently and sharply locally at variation amplitude of 200 and lower. Distance between the smallest mean and the best individual is medium compared with the previous value. The distance between the mean and best individual converges. The mean aggregates quickly to 288.4 over five generations, then much closer to the best individual, gradually decreasing over many generations similar to previous optimizations.

The objective function value $(0.706)$ corresponding to the best individual is distinctly less than 13.441 and 2.27 of the previous optimizations.

For the situation covering minimum $\mathrm{AB}$, parameters $n=2, l=3$ and $k=2$ are substituted in eq. (2) to update parameter error $_{i}$ in the new objective function, in which 11 variables need to be estimated numerically.

Figure $1 d$ shows the relation between the new function and crossover fractions. All values can be divided into three groups: one is larger between 1 and 1.7, another between 0.7 and 0.3 , and the last is the least value including 2 points separated and unstable. The function value reaches close to its minimum more gradually at the cross- over fraction of 0.3 than at 0.7 ; so 0.3 is selected as the proper crossover fraction for its relatively stable function value. The objective function values are generally not stable, similar to the situation of the four CSPs.

The convergence process of the third optimization for the five CSP situation shown in Figure $2 d$ is similar to that for the four CSP. It shows higher diversity in the front segment and good convergence of individuals thereafter. Compared with the four CSP, the largest mean, as large as $1.47 \times 10^{4}$, has the same order of magnitude signifying good generation diversity. The distance converges and the mean value aggregates quickly to 281.4 over five generations and gradually slows down, which is similar to the four CSP optimization. The mean in the middle iteration fluctuates inconsistently, sharply locally at variation amplitude of 200 and more frequently than that of the four CSP situation. Distance between the smallest mean and best individual is much closer than that of the four CSP situation, but a little large.

Generations (thousands) in the two situations for four CSP and five CSP are much more than those (about several hundred) in previous optimizations. Table 4 lists some control parameters and calculation results for these two situations.

According to the third optimization for four and five CSPs, the final mean objective-function values are 0.0706 and 0.0725 respectively, much lower than DP of $0.1^{\circ}$. Thus, all CSPs are considered to have been ascertained by this hybrid GA, and the optimization process should end. In this case, three major CSPs and two minor CSPs develop and affect the reservoir-abutment safety to different extents. The locations of major CSPs (from detection points \#10 to \#1) are 39.8, 179 and $139 \mathrm{~m}$, whereas the minor ones are located at 220 and $346 \mathrm{~m}$.

The maximum values of objective functions changing with crossover fractions decrease gradually from about $17,5.0$ to 2.5 and 1.7. The minimum also decreases gradually from about $13.4,2.6$ to 0.1 , and the individual variation range has the same tendency from 3.6 to 0.8 almost at the same pace. The objective function continuity becomes poor from the first to the last step. More local minimums of the objective function are isolated, and the result is more unstable. 


\section{RESEARCH COMMUNICATIONS}

According to actual optimization steps, the objective function value decreases from 13.44, 2.27 to 0.7 from the first to the last optimization. All the iterations converge distinctly and rapidly. Individuals in all optimizations are diversified for the selected large population and the large magnitude of the objective function. After a rapid decrease, convergence has different characteristics. The constantly changing current individuals indicate many local optima.

The coefficient values imply the seepage intensities of CSPs. The largest, $\beta_{22}$, indicates that the first CSP has the strongest seepage intensity; the second largest, $\beta_{23}$, is about 0.16 times the largest; the third largest, $\beta_{24}$, is about 0.5 times the second. The second smallest is $\beta_{25}$, about 0.1 times the third largest, and much smaller than the three larger CSPs. The smallest $\beta_{26}$, is about 0.3 times the second smallest and significantly smaller than the others.

As impact from weak intensive CSPs on the right side are eliminated, the first CSP location continuously decreases from 60.829 to 41.256 to 39.86 during optimization. The final location is farther from point \#10 than 38.66 by another method ${ }^{13}$. The initial second CSP location (172.59) from this method is also larger than that (170.53) from $\mathrm{SA}^{15}$, but increases (179.8) when the effect from minor CSP on its left is eliminated. The location (139.4) of the third CSP is also greater than 99.2 by SA ${ }^{15}$, and locations 220 and 346 of the fourth and fifth CSPs are significantly smaller than 260 and 367, with a larger objective function value of 1.89 by other methods ${ }^{13}$.

Influences from the fifth CSP, with the smallest seepage intensity on other CSP locations are not that effective as on the coefficients, since the absolute per cent relative differences of the locations between the two situations are no more than $0.18 \%$ and the absolute per cent relative differences of coefficients between the two situations are from $0.63 \%$ to $37.44 \%$. The fifth CSP has significant impact on the constant, $\beta_{21}$, in one order of magnitude difference, and more on $\beta_{25}$. Among the effects on locations, the fifth CSP affects the first location most and the fourth least almost without variation.

Based on the optimization results, some conclusions can be drawn:

The first CSP, about $39.8 \mathrm{~m}$ from detection point \#10, has the most significant impact on abutment safety; the one about $139 \mathrm{~m}$ from point \#10 has the second most significant impact, and the third one is at $179.3 \mathrm{~m}$. These are the three principal causes of water loss of the reservoir and abutment erosion. These concentrated seepage should be cut off as early as possible. The two minor ones have a slight impact on dam safety and water leakage, and need to be treated to prevent them from developing into larger ones.

In contrast with previous studies ${ }^{1,7,8,13,16}$, optimization trial amount decreases because of specified crossover fractions. The approximate crossover values can be obtained according to the minimum and stability of objective function values depending on crossover fractions.
Implementing 'Fmincon' effectively diminishes the objective function values from GA to different extents, which implies 'Fmincon' can being the optimization results closer to the global optimal.

Initial value constraints by $\mathrm{AB}$ accelerate the optimization and reduce the computation duration, as verified by computation trial contrast. It is sound that the relative coefficient sizes are constrained according to the physical meanings of CSP seepage intensity.

The full-parameter hybrid optimization virtually eliminates impacts by smaller CSPs on the longer ones. The resultant coefficients and locations of CSPs are closer to the real-world.

It is feasible for practical applications to locate multipassage seepage by this full-parameter hybrid GA. Combining sub-step residual analysis and one-off solving eliminates the smaller CSP impact on higher seepageintense CSPs. Moreover, results are more accurate, and the process is more concise and easily integrated.

1. Wang, X. J. and Pan, J. S., Location detection of concentratedseepage passages in dam by groundwater temperature. Chin. J. Geotech. Eng., 2010, 32(11), 368-374.

2. Dong, H. Z., Liu, Y. X. and Zhang, Y., Finite long heat source model of piping in dam and experiment. Chin. J. Hydraul., 2012, 43(8), 1004-1008.

3. Dong, H. Z., Lou, R. H. and Zhang, L., Study on doubleconcentrated-leakage passage heat conduction model and retrieval of velocity in dam basement. J. Sichuan Univ., 2012, 44(3), 3641 .

4. Dong, H. Z., Kou, D. W. and Peng, H. Y., Computational model for dam leakage velocity in concentrated passage based on distributed optic fiber temperature sensing system. Chin. J. Geotech. Eng., 2013, 35(9), 1717-1721.

5. Dong, H. Z. and Zhang, X. Y., Seepage cylindrical heat source model of dam and experimental study. Chin. J. Rock Mech. Eng., 2011, 30(s2), 3665-3671.

6. Wang, X. J. and Chen, J. S., Research of temperature tracer method to detect tubular leakage passage in earth-dam. J. China Univ. Min. Technol., 2006, 16(3), 353-358

7. Wang, X. J. and Li, R. Y., Research on detecting multi-passage leakage in dam by temperature in bores. Adv. Mater. Res., 2012, 46-49, 1959-1962.

8. Wang, X. J., Li, R. Y. and Chen, J. S., Concentrated-leakage detection by emulation of temperature field. Appl. Mech. Mater., 2012, 224, 93-96.

9. Chen, J. S., Fan, Z. C. and Dong, H. Z., Detecting the damdetouring seepage passage in the left dam abutment of douhe reservoir with the method of heat source. Hydrogeol. Eng. Geol., 2006, 5, 61-65.

10. Dong, H. Z. and Chen, J. S., Model research of heat source method by using water temperature distribution in borehole to determine seepage velocity of dyke. Hydrogeol. Eng. Geol., 2003, $5,40-43$.

11. Chen, L., Wu, Y. and Chen, J. S., Study on temperature field inverse analysis and experiment of concentrated seepage passage in dam base. J. East China Inst. Technol., 2007, 30(4), 354-257.

12. Wang, X. J., Tong, H. B. R. and Li, Y., Locating the plane concentrated seepage in dam by transient temperature field. In Progress on Mine Safety Science and Engineering, First Int. Symposium Mine Safety Science and Engineering A, Beijing, 2011, pp. $1749-1755$. 
13. Wang, X. J., Zhu, D. L. and Pan, J. S., Locating concentrated seepage pipes in dam with temperature global optimization. J. Eng. Geol., 2015, 23(2), 335-343.

14. Wang, X. J., Li, R. Y. and Wei, S. M., Substep simulated annealing to locate multiple concentrated seepage passages in dams using ground temperature. E-J. Geotech. Eng., 2015, 20(15), 6603-6614.

15. Abu-Reesh, M. I. and Alnaizy, R., Optimal design of multi-stage bioreactors performing wastewater treatment using the MATLAB optimization. Int. J. Environ. Eng., 2015, 6(4), 403-415.

16. Dadashi, E., Ahangari, K., Noorzad, A. and Arab, A., Support system suggestion based on back-analysis results case study: Babolak water conveyance tunnel. Arab. J. Geosci., 2012, 5(6), $1297-$ 1306.

17. Fernandes, F. P., Costa, M. F. P. and Fernandes, E. G. P., A derivative-free filter driven multistart technique for global optimization. Comput. Sci. Appl., Lecture Notes Comput. Sci., 2012, 7335, 103-118.

18. Takbiri, Z. and Afshar, A., Multi-objective optimization of Fusegates system under hydrologic uncertainties. Water Resour. Manage., 2012, 26(8), 2323-2345.

ACKNOWLEDGEMENTS. This study was supported by China Scholarship Council (201308410082); the National Natural Science Fund Project (41174052508902) and Henan Province and Frontier Technology Research Projects (1123004024), China.

\section{Identification of begomoviruses from legume crop and weed plants and viruliferous status of the whitefly Bemisia tabaci in Central India}

\author{
Rakesh Singh Marabi ${ }^{1, *}$, Shoumitra Bikash Das ${ }^{1}$, \\ Niraj Tripathi ${ }^{2}$, Takashi Wada ${ }^{3}$ and \\ Hiroaki Noda ${ }^{3,4}$ \\ ${ }^{1}$ Department of Entomology, College of Agriculture, \\ Jabalpur 482 004, India \\ ${ }^{2}$ Directorate of Research Services, Jawaharlal Nehru Krishi Vishwa \\ Vidyalaya, Jabalpur 482 004, India \\ ${ }^{3}$ Japan International Cooperation Agency Soybean Project, \\ ADR Building Research Campus, College of Agriculture, \\ Indore 452 001, India \\ ${ }^{4}$ National Institute of Agrobiological Sciences, Owashi, \\ Ibaraki 305-8634, Japan
}

Three begomoviruses were identified based on complete genome sequence from weeds in Jabalpur, Madhya Pradesh, Central India. Alternanthera yellow vein virus (AIYVV; 2745 bp) was identified in Alter-

\footnotetext{
*For correspondence. (e-mail: rsmarabi78@rediffmail.com)
}

nanthera sessilis, Tobacco curly shoot virus (TbCSV; 2766 bp) in Sida acuta, and Cotton Leaf curl Bangalore virus (CLCuBaV; 2750 bp) in Malvastrum coromandelianum. A betasatellite DNA (1356 bp) was found associated with $\mathrm{CLCuBaV}$, sharing sequence identity of $87-88 \%$ with those of other CLCuBaV isolates. TbCSV was detected in the food legumes soybean, black gram and pigeon pea, showing coinfection with Mungbean yellow mosaic India virus (MYMIV). Whitefly Bemisia tabaci, the vector of begomoviruses, harboured TbCSV and CLCuBaV at a high rate $(10-60 \%$ and $5-30 \%$ respectively). The percentage of insects harbouring MYMIV was also extremely high (60-100), indicating that the whiteflies were viruliferous for multiple begomoviruses in this region. High population density of the whitefly and intensive cultivation of crop plants promote mixed infection of various begomoviruses in the fields of Central India.

Keywords: Begomovirus, Bemisia tabaci, legumes crops, viruliferous status, weeds.

GEMINIVIRUSES (family Geminiviridae) are singlestranded DNA (ssDNA) viruses causing severe economic losses to agricultural production worldwide ${ }^{1}$. Geminiviruses possess a genome comprised of one or two circular ssDNA molecules of $2.5-2.8 \mathrm{~kb}$ (refs 2, 3). They exhibit considerable diversity in terms of their genome structure, and are presently classified into nine genera ${ }^{4,5}$. Begomovirus is the largest genus in family Geminiviridae that includes more than 320 begomovirus species ${ }^{5}$. Many of the begomoviruses consist of two genome components, referred to as DNA-A and DNA-B ${ }^{2,6}$. Several begomoviruses were identified to have only a single component equivalent to DNA-A. However, some begomoviruses instead need another circular ssDNA component of approximately half the size of DNA-A to induce typical disease symptoms in their hosts ${ }^{3,7}$.

Begomoviruses are transmitted by the whitefly Bemisia tabaci Gennadius (Hemiptera: Aleyrodidae). This is a species complex ${ }^{8}$ and more than 35 putative species are included $^{9-12}$. The whitefly, which infests many plant species, transmits begomoviruses to various plants ${ }^{13}$. Begomoviruses are transmitted by whitefly in different crop plants in a circulative manner ${ }^{14,15}$. In Central India, $B$. tabaci is characterized as Asia I and II genetic groups ${ }^{16}$, whose population increases in the summer season (AprilJune), and viruliferous whiteflies migrate into the fields of soybean that is a major crop in the kharif season (June-September). Population density of the whitefly is generally extremely high on legume plants and weeds in the fields of Central India in this season ${ }^{17}$.

Mungbean yellow mosaic India virus (MYMIV), a member of the genus Begomovirus, causes yellow mosaic disease (YMD) in legume plants, and is a threat for important food legumes such as soybean (Glycine max), 